\title{
HIP-Storytelling: Hand Interactive Projection for Storytelling
}

\author{
Narciso Melo ${ }^{1}$, Pedro Salgado ${ }^{1}$, Ido Iurgel ${ }^{123}$, Pedro Branco ${ }^{13}$ \\ ${ }^{1}$ engageLab, University of Minho, Guimarães, Portugal \\ \{narciso.melo, pedro.salgado\}@engagelab.org \\ ${ }^{2} \mathrm{CCG}$ (Computer Graphics Center), University of Minho, Guimarães, Portugal \\ iiurgel@ccg.pt \\ ${ }^{3}$ Department of Information Systems., University of Minho, Guimarães, Portugal \\ pbranco@dsi.uminho.pt
}

\begin{abstract}
We have created an interactive storytelling system for public spaces that is collaborative, easy and entertaining to use, and allows for a natural interaction. The system consists of a table, a ceiling mounted projector that projects onto the table, and a 3D camera for tracking hands and for object recognition. The main feature of the system is the projection on top of the palm of the hand of the users; thus, the hand becomes also a viewing surface. This allows for very natural gestural interaction, such as holding and passing objects between users. For example, in the course of narrative, users can hand over a story character or object to each other. We also employ projection based augmented reality to animate real objects on the table. Apart from entertainment, the system shall be employed for concrete educational interactive storytelling applications in public spaces.
\end{abstract}

Keywords: Interactive Storytelling, HCI, Natural User Interfaces, Augmented Reality.

\section{Introduction: Interactive System at Public Spaces}

What is the best way to employ interactive storytelling for practical, fail-safe applications in public spaces, possibly with educational goals, e.g. for kids? We have created an interactive storytelling system for public spaces such as museums or shopping malls where interactive storytelling can be presented in an innovative, funny and engaging way, and where users can collaborate with each other. Our system shall also enable to transmit knowledge in an entertaining way, e.g. in museum contexts. In place of the widespread "point \& click" interfaces and of touch surfaces, in our system, the user interacts with the narrative through natural hand movements and gestures. Using the recent of-the-shelf $3 \mathrm{~d}$ cameras and computer vision algorithms, we track objects and hand palms, and project narrative elements onto the hand and the objects, enabling gestures such as picking up some virtual object or dropping it, and 
specifically collaboration, e.g. handing over a virtual, projected object to another user. ${ }^{1}$

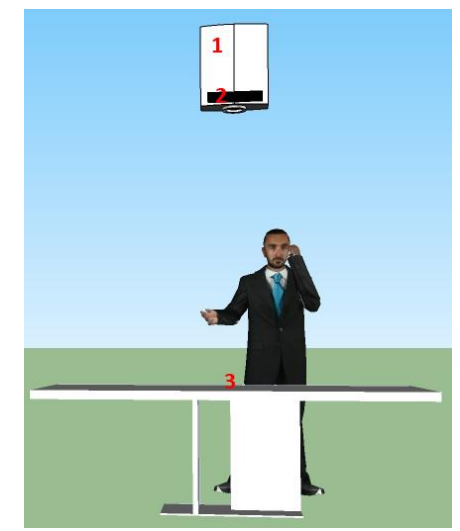

Fig. 1. Configuration of the system (1-projector, 2-3D camera, 3-projection surface, e.g. table)

\section{Related Work}

Most interactive systems for public spaces today are based on touch or multitouch screens. With this project we aim to design interactions that are more natural and appropriate for the content and for intended usage. Ishii and Homura have presented the artwork "it's fire, you can touch it" [1] in which the palm of the hand is employed as projection surface for poems. This system was a major inspiration of our work. We take the concept further by transforming this palm-projection idea into the main mode of interaction, allowing for collaboration through natural gestures. In future, more types of gestures shall be allowed for.

There are several examples of previous work merging augmented reality systems that project directly on a table top with the manipulation of physical objects placed on that surface, in the authors opinion a particular interesting example is the OASIS project [2] as it recognizes a variety of objects placed on the table and where the virtual objects react to the physical objects.

The Responsive Multimedia System (RMS) [3] is an example of a tangible system designed for interactive storytelling. Users can interact with tangible objects to experience a personalized interactive story. The table in RMS is used as tangible user interface consisting in a semi-transparent display screen. A camera tracker is placed under the table to recognize objects and touch.

\footnotetext{
${ }^{1}$ Another practical advantage of the system is that it is hygienic, since interaction can be completely touchless, which is an important consideration for public spaces.
} 
Another previous work coupling tangible interfaces with a storytelling system is the project "Tangible Viewpoints" [4]. It explores a multimedia storytelling system that uses a tangible interface to represent different character perspectives for the story. The interaction surface on which the objects are placed is complemented by a LCD screen with speakers where the action is presented.

The HIP system here presented consists of a table, a ceiling mounted projector that projects onto the table, and a 3D camera for tracking hands objects. Objects placed on the table can be augmented while the users' hand serves both as a localized display and as an interaction mechanism. Multiple users can interact simultaneously with the scene or in-between users allowing for example to grab and exchange objects. Given the naturalness of the gestures and movements we believe this provides for a quite intuitive and immersive interaction since the virtual character and objects are not confined to a screen; users have informally reported that a tactile sensation is invoked when an image of an object is projected onto the surface of their palm, which adds a tangible presence to the virtual thing (Cf. [1] for a similar report).

\section{$3 \quad$ HIP-Storytelling}

We have currently implemented and tested the hand recognition and projection of HIP-Storytelling, and our first successful test case has been a fish tank. Users can grab fishes from it, carry them on their palms, and hand them over to other visitors, cf. Figure 2. We are currently working on an interactive storytelling prototype for edutainment in museums. It shall transmit an important event in the history of Portugal. The story logic is not in focus at this moment, and we will employ a simple "string-of-pearls" approach (cf. [5]), where users have a certain level of freedom to solve some tasks at certain times during the narrative, but it still follows a linear storyline.

Story characters will be projected onto the table; users will interact with them with the hand, picking, dropping and carrying them, and handing them over to others.

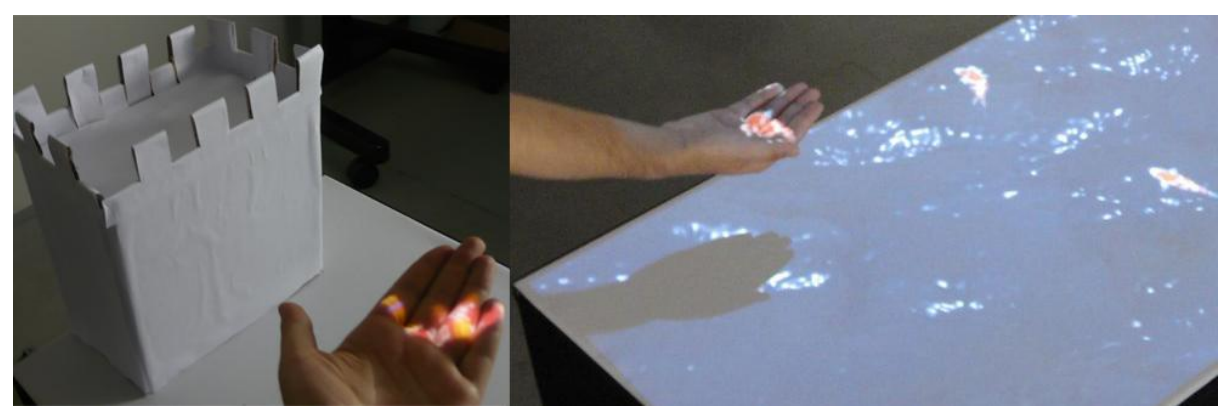

Fig. 2. First test case with fish tank and a current working on test with a real object (castle) 
Our prototype shall tell an interactive story based on the history of Portugal and the city of Guimarães, about a battle that Dom Afonso Henriques fought against his own mother (and which he won). Users must accomplish tasks to progress within the narrative. For example, placing armies on the battlefield, helping Dom Afonso Henriques to fight or to climb into the castle; users can also grab enemies and throw them to another location. Some tasks are easier to cope with if several users collaborate, e.g. an easy way to hide the mother from the son is for one visitor to hold her in his hand, while the other user carries the son to the castle. It shall also be possible to interact with real objects, such as the castle and catapults; these tangible elements will be recognized by computer vision algorithms, they shall be part of an Augmented Reality setting, in the sense that the objects will be augmented will be projected by projections on top of them, for instance fire on the castle or bullets on the catapults.

\section{Conclusion}

At first informal tests, some users reported a series of tactile sensations when viewing objects (fishes) projected into their hands. This phenomenon of synesthesia is compatible with Biocca et al. [6] findings, and contributes to a sensation of immersion and liveliness. We believe that HIP-Storytelling is an important example of how interactive storytelling can be employed in public spaces, where immediate understanding of the interfaces, collaboration, and a sense of fun and involvement are important.

\section{References}

1. Ishii, Y., Homura, H.: It's fire, you can touch it: http://www.okcentrum.at/?q=en/content/presse/701: July 5, 2011

2. OASIS (Object-Aware Situated Interactive System), http://software.intel.com/enus/videos/oasis-object-aware-situated-interactive-system/: July 5, 2011

3. Lee, Y., Oh, S., Park, Y., Woo, W.: Responsive Multimedia System (RMS) for Personalized Storytelling: Proceedings of The 4th international symposium on ubiquitous VR: 2006, pp. 29-32

4. Mazalek, A., Davenport, G., Ishii, H.: Tangible Viewpoints: a Physical Approach to Multimedia Stories: Proceedings of the 10th ACM international conference on Multimedia (MM 2002): 2002, pp. 153-160

5. Majewski, J.:THEORISING VIDEO GAME NARRATIVE: 2003: Master: Bond University

6. Biocca, F., Kim, J., Choi, Y. : Visual Touch In virtual environments: An exploratory study of presence, multimodal interfaces, and cross-modal sensory illusions.: Presence, Vol. 10, No. 3: 2001, pp. 247-265 